\title{
Contribution à l'étude des Ixodidae (Acarina, Ixodoidea) des Monts Cantabriques (Espagne)
}

\author{
par B. GILOT *, G. PAUTOU **, J. GOSALBEZ *** et E. MONCADA \\ * Laboratoire de Parasitologie, Faculté de Médecine (U.E.R. Santé dans la Collectivité) \\ Avenue du Professeur Léon-Bernard, F 35000 Rennes \\ ** Laboratoire de Biologie végétale, Université Scientifique et Médicale de Grenoble, \\ Domaine Universitaire, B.P. 53, F. 38041 Grenoble Cedex. \\ *** Departamento de Zoologia, Facultad de Ciencias, \\ Universidad de Barcelona, Espagne.
}

\section{Résumé.}

Cinq espèces d'Ixodidae parasites des micromammifères ont été identifiées dans le NordOuest de l'Espagne, plus particulièrement dans les Monts Cantabriques.

Parmi ces espèces, les auteurs soulignent la présence d'Ixodes acuminatus (Neumann, 1901) et d'Ixodes trianguliceps (Birula, 1895) qui, à leur connaissance, n’ont jamais été signalés dans la Péninsule Ibérique.

\section{Summary.} (Spain).

Contribution to study of Ixodidae (Acarina, Ixodoidea) in Cantabric Mountains

Five Ixodidae species parasites of small mammals have been identified in North-western Spain and particularly on the cantabrique Mountains.

The authors mention that among these species there are Ixodes acuminatus (Neumann, 1901) and Ixodes trianguliceps (Birula, 1895), which according to their own knowledge, have never been noticed in the Iberian Peninsula so far.

* Attaché de Recherches à l'I.N.S.E.R.M.

Annales de Parasitologie humaine et comparée (Paris), t. $51, \mathrm{n}^{\circ} 2$ 


\section{Introduction}

Les tiques de la Péninsule Ibérique ont fait l'objet des travaux de base de Gil Collado (1936, 1938, 1948). Plus récemment, Acedo et al. (1973), étudiant les ectoparasites de mammifères et d'oiseaux du Haut Aragon, citent un certain nombre d'espèces de tiques dont les hôtes sont représentés par des mammifères de grande taille.

Cependant, aucun des travaux que nous venons d'indiquer ne fait état de captures de tiques sur les micromammifères: c'est cette lacune que nous allons essayer de combler en étudiant, dans le cadre de plusieurs publications, l'abondant matériel prélevé au cours de quatre missions effectuées de 1970 à 1973 dans la Péninsule Ibérique, missions qui nous ont permis d'identifier la faune ixodidienne de plus de 1000 micromammifères; à ce matériel s'ajoutent les récoltes effectuées par l'un de nous, en diverses régions de l'Espagne, notamment au cours des années 1973 et 1974.

Notre première contribution s'appuie sur le matériel que nous avons nous-mêmes collecté au cours de la mission de 1973, centrée sur le Nord-Ouest de l'Espagne. Cette expédition (16 septembre-4 octobre 1973) nous a permis d'examiner près de 400 micromammifères et de récolter plus de 200 tiques sur un peu plus du quart des animaux capturés. Ces micromammifères ont été piégés aux environs de 9 stations principales situées dans les provinces de Santander, Oviedo, Leon, Lugo, Orense, Zamora. Cependant, la majorité de nos récoltes ayant été effectuée dans les Monts Cantabriques et les zones de basse altitude avoisinantes, c'est cette chaîne montagneuse et son avantpays qui formeront l'ossature de notre travail.

\section{I. - Méthode}

Rappelons, d'abord, que les tiques qu'on peut rencontrer sur micromammifères (rongeurs, insectivores, petits carnivores) peuvent en fait avoir deux significations différentes: ou bien il s'agit de tiques dites «spécifiques » de ces micromammifères qui en sont les hôtes exclusifs (tel est le cas, par exemple d'Ixodes trianguliceps dont tous les stades parasitent les micromammifères) ; ou bien il s'agit exclusivement d'immatures (larves et nymphes) appartenant à des espèces exophiles dont les adultes parasitent des mammifères de grande taille ; tel est, notamment, le cas des espèces suivantes: Ixodes ricinus (Linné, 1758), Ixodes ventalloi (Gil Collado, 1936), Dermacentor marginatus (Sulzer, 1776), Dermacentor reticulatus (Fabricius, 1794), Rhipicephalus turanicus (Pomerantsev, Matikaschvili, Lototzki, 1940). Pour de telles espèces, l'étude des immatures ne devrait intervenir que pour servir de complèment à d'autres démarches : recherche des tiques adultes sur grands mammifères, recherche des tiques libres sur la végétation par la méthode du «drapeau»; si cette recherche globale n'a pu être effectuée dans le cadre d'une expédition dont le but principal était la capture des micromammifères, quelques collectes sur animaux domestiques ou même «au drapeau» ont pu être réalisées, qui ont complété les informations obtenues par notre méthode de base. 
A vrai dire, l'échantillonnage que nous avons tenté de réaliser est loin d'être parfait: les critiques qu'on peut lui adresser proviennent principalement de trois raisons :

- Le grand nombre de pièges que nous avons mis en œuvre pour multiplier les captures a une contrepartie défavorable parce que plusieurs modèles différents ont été employés. C'est manifestement la «Longworth mammal trap » qui s'est révélée la plus adaptée à cette étude : ce piège permet en effet la collecte des tiques dans les meilleures conditions (le micromammifère étant capturé vivant, dans la plupart des cas, le parasite est décelé, bien souvent, alors qu'il est encore fixé sur son hôte ; si la tique s'est détachée, parce que son degré de gorgement est suffisant, elle est aisément retrouvée lorsqu'on examine la «litière » dont on a pris soin de garnir le piège). Or, aux côtés de ce piège «idéal », des pièges d'autres types ont été employés: piègestrappes ou pièges-couloirs du type I.N.R.A., dont l'efficacité, pour la capture des ectoparasites, est beaucoup plus incertaine.

- L'investigation des différents milieux n'a pas été toujours égale: certains milieux jugés a priori pauvres en micromammifères, les Chênaies à Quercus ilex par exemple, ont été souvent négligés au bénéfice de milieux plus productifs.

- Le choix de la date d'expédition a certainement influencé grandement nos résultats. Certes, un tel choix est difficile à prendre a priori lorsqu'il s'agit d'une région vierge de toute investigation: la phénologie des différentes espèces de tiques n'est pas exactement la même d'un pays à l'autre, même s'il existe certaines constantes. De toutes façons, dans une expédition de courte durée, certaines espèces risquent d'être défavorisées par rapport à d'autres.

Remarquons toutefois que, si la date a été retenue avec un peu d'arbitraire, cette période de début d'automne nous a paru, en définitive, assez favorable pour la plupart des espèces.

Cela est vrai, notamment, pour les tiques du genre Dermacentor ou Rhipicephalus. Ainsi, nos récoltes en France d'immatures de Dermacentor (plus particulièrement ceux de $\nu$. marginatus) se situent surtout en été, mais également en début d'automne. Les récoltes que nous avons effectuées nous-mêmes dans d'autres régions d'Espagne montrent qu'il en est exactement de même pour $R$. turanicus, dont la période d'activité maximale des immatures est située en été ; cependant les nymphes de cette espèce se rencontrent encore au mois de septembre et même au début du mois d'octobre.

En ce qui concerne le genre Ixodes, nous considérerons d'abord le cas des espèces non spécifiques: Ixodes ricinus et Ixodes ventalloi. Les seules données que nous ayons sur l'activité des phases immatures d'Ixodes ricinus en domaine atlantico-européen se rapportent à vrai dire à une région fort éloignée de celle qui nous intéresse ici : le Brionnais, en France (Simon, 1974). L'activité larvaire, notamment, y est forte en cette période de début d'automne. Tout ce qu'on connaît sur l'activité des immatures d'Ixodes ventalloi se trouve dans les travaux de Blanc et Bruneau (1958) sur le Maroc: si le printemps paraît représenter la période la plus favorable pour l'activité larvaire, le mois d'octobre marque une recrudescence de cette activité après la chute estivale. 
Le dynamisme saisonnier des tiques spécifiques commence à être connu grâce aux nombreuses récoltes effectuées en France. Ainsi, en ce qui concerne Ixodes trianguliceps, les captures effectuées dans diverses régions de France montrent que l'activité de cette espèce peut être constatée toute l'année: le début de l'automne est une période très favorable pour les adultes (Gilot et al., à paraître). De même, les femelles d'Ixodes acuminatus sont trouvées facilement en automne, notamment sous climat atlantique. Pour ce qui est des immatures, Morel (1965) constate sur les mulots des Pyrénées-Orientales « une activité larvaire et nymphale qui s'étale de mai à septembre ».

Quoi qu'il en soit, malgré les critiques qu'on peut formuler contre notre échantillonnage, les résultats de notre étude nous paraissent intéressants à un double titre : intérêt biogéographique général, puisqu'elle nous permet de signaler, pour la première fois en Espagne, deux espèces qui n'y étaient pas connues: Ixodes trianguliceps et Ixodes acuminatus; intérêt écologique également, puisqu'elle nous a permis d'étudier un certain nombre de biotopes pour cinq espèces de tiques et de comparer ces biotopes avec ceux que nous connaissons en France.

\section{II. - Le milieu}

Les prospections ont été surtout effectuées dans le Massif des Picos de Europa, qui se situe approximativement au milieu de la chaîne cantabrique. Ce massif, dont les plus hauts sommets atteignent 2700 mètres, présente une ossature vigoureuse de roches calcaires, sur laquelle s'appuient des affleurements de schistes et de grès qui sont à l'origine de reliefs plus mous donnant un aspect vallonné aux parties inférieures.

Par suite de sa situation à l'extrémité septentrionale de la Péninsule Ibérique, le massif est soumis à un climat général très humide. Gijon, situé sur la côte cantabrique à une cinquantaine de kilomètres du massif, reçoit $1000 \mathrm{~mm}$ de pluie. Les précipitations sont assez régulièrement réparties sur toute l'année, novembre et décembre étant les mois les plus arrosés. Il n'y a pas de période sèche. C'est un climat doux, à faible amplitude thermique (température moyenne annuelle de Gijon: $13,7^{\circ} \mathrm{C}$; température du mois le plus chaud: $19,1^{\circ} \mathrm{C}$; température du mois le plus froid : $9,4^{\circ} \mathrm{C}$, d'après Valenti, 1968). Dans son étude sur les ensembles climatiques de la Péninsule Ibérique, Gaussen (1957) classe ce climat dans les climats hypomésaxériques. Avec l'altitude, les précipitations augmentent rapidement. Elles dépassent $1500 \mathrm{~mm}$ au-dessus de $1200 \mathrm{~m}$ et atteignent vraisemblablement $2000 \mathrm{~mm}$ dans les parties les plus arrosées. Ces totaux pluviométriques sont en fait du même ordre que ceux des massifs bien arrosés des Pyrénées ou des Alpes. Les étages montagnard et subalpin se caractérisent par un hiver qui dure plusieurs mois (climat axérique froid de montagne, d'après Gaussen). Cependant l'orientation générale O.-E. de la chaîne prédispose les versants nord et sud à des conditions climatiques très contrastées. Le versant septentrional soumis aux influences océaniques est copieusement arrosé par les pluies apportées par les vents d'ouest et les vents du nord ; ces derniers sont à l'origine des chutes de neige en hiver. Le versant méridional, en revanche, est soumis aux influences continentales de la meseta 
qui se manifestent par des vents du sud très secs. Ces conditions climatiques expliquent le recouvrement considérable des hêtraies en versant nord où elles descendent jusqu'à 700 mètres d'altitude. Le Hêtre est beaucoup plus rare en versant sud, où il ne constitue véritablment des peuplements purs qu'à la faveur de conditions microclimatiques favorables.

L'analyse de la végétation est, comme nous l'avons montré (Gilot et al., Acta tropica, 1976) une approche indispensable pour la recherche des populations d'Ixodidés. Aussi l'étude géobotanique de Lascombes (1944) constitue-t-elle un document fondamental pour interpréter nos résultats.

Les vallées, les différents bassins sont intensément cultivés: les champs, les prairies et les vignes donnent un aspect mosaïqué aux paysages qui entourent les villages. La végétation naturelle est cependant bien représentée sur les sols peu favorables ou impropres à la culture. Depuis les parties inférieures jusqu'aux sommets, on peut distinguer :

a) Les Chênaies à Quercus ilex, qui sont surtout bien représentées sur le versant sud.

b) Les Chênaies à Quercus lusitanica, qui sont peu fréquentes. Ce Chêne semble rechercher les situations les plus fraîches des parties inférieures du massif. Son cortège est constitué par des espèces mésophiles et mésoxérophiles qui trouvent des conditions optimales dans les Chênaies caducifoliées.

c) Les Chênaies à Quercus petraea et $Q$. tauza: elles s'installent sur les sols aciảes provenant de la décomposition des schistes. Sur le versant sud, elles atteignent 1000 mètres aux expositions les plus favorables. Le cortège floristique comporte des mésophiles et des méso-hygrophiles des sols acides: Erica cinerea, Genista pilosa, Sarothamnus cantabricus, S. scoparius. Les groupements de dégradation les plus communs sont les landes à Pteris aquilina, les landes à Ericacées et Papilionacées, les fourrés de Corylus avellana.

d) Les Chênaies à Quercus pedunculata, à cortège floristique composite, doivent être bien représentées dans les parties inférieures du versant nord. L'auteur ne donne pas d'informations précises sur leur composition floristique et sur leur importance quantitative.

e) Les Hêtraies. Elles constituent le groupe climacique de l'étage montagnard humide, où on peut les trouver entre 700 et 1400 mètres. Le cortège floristique est souvent très pauvre. Il comporte un grand nombre d'espèces présentes dans les Hêtraies mésophiles et les Chênaies à Charme des Alpes. Ces Hêtraies constituent un milieu privilégié pour l'installation des tiques.

Les landes se substituent souvent au Hêtre, lorsque les conditions sont défavorables à l'espèce (vents violents). Parmi les groupements de dégradation de la série, il faut citer les landes à Ericacées, les landes à Genêts, les frutiçaies à Erica arborea; des prairies méso-hygrophiles, où on retrouve plusieurs espèces des mégaphorbiaies, s'instalient sur les sols à forte rétention hydrique. 
A côté de ces groupements qui constituent l'essentiel des peuplements forestiers, il faut signaler deux groupements qui sont également favorables à l'installation des Ixodidés :

- les Châtaigneraies qui, de place en place, semblent constituer des faciès de substitution des Chênaies caducifoliées ;

- les forêts riveraines à Alnus glutinosa. Ce groupement compte de nombreuses espèces qui existent dans les forêts en bordure de ruisseaux qui descendent du Jura ou des Préalpes: Acer campestre, A. platanoides, Tilia parvifolia, T. platyphyllos, Juglans regia.

La limite supérieure de la forêt, qui se situe vers 1400 mètres, apparaît très basse quand on la compare à celle des massifs pyrénéens ou alpins. Nous ne chercherons pas d'explication et nous renvoyons le lecteur aux interprétations de Lascombes. Nous la constatons simplement et considérons que c'est un fait important.

Il est vraisemblable que les groupements herbacés qui colonisent les parties supérieures du massif sont à rattacher à plusieurs étages de végétation : étage montagnard, subalpin et alpin. Dans les parties inférieures, on retrouve les groupements présents dans l'étage du Hêtre. Dans les parties plus nettement subalpines, s'installent des landes à Vaccinium myrtillus, des landes à Juniperus nana. Les pelouses à Festuca rubra, les pelouses à Festuca ovina, Sesleria coerulea sont également bien représentées.

De cette analyse sommaire de la végétation, nous ferons trois remarques:

- Les parties sommitiales dépourvues de végétation arborescente sont favorables à la vie pastorale et soumises à une intense pression pastorale en été. Les migrations saisonnières du bétail sont fondamentales pour comprendre la distribution verticale des Ixodidés.

- On constate que les landes sont très bien représentées et nous rappellerons que ces milieux sont particulièrement favorables à l'installation des tiques du genre Dermacentor.

- Il faut souligner enfin les surfaces considérables occupées par des groupements forestiers (Hêtraies) liés à une humidité atmosphérique élevée, dont nos recherches dans les Alpes nous ont montré qu'ils étaient favorables à divers Ixodes (Ixodes ricinus, Ixodes trianguliceps).

\section{III. - Les espèces}

\section{A) Tiques exophiles.}

Nous entendons par là les tiques dont les adultes pratiquent couramment l'exophilie. Les immatures de ces mêmes espèces ont un comportement soit totalement soit partiellement endophile. 
1) IXODES RICINUS. - Cette espèce apparaît de loin comme l'espèce la plus abondante dans nos échantillons. Elle est présente depuis les vallées (180 mètres) jusqu'à 1200 mètres. Nous ne l'avons pas trouvée au-delà ; il est vrai que nous n'avons échantillonné que peu de milieux entre 1200 et 1800 mètres.

Ixodes ricinus est particulièrement bien représentée dans les Hêtraies. Nous rappellerons que, dans les Alpes françaises, l'espèce trouve des conditions optimales dans les groupements mésophiles de l'étage collinéen et du Montagnard inférieur (Gilot et al., Acta tropica, 1975): les Chênaies à Charme sont particulièrement favorables, et il est intéressant de constater que de nombreuses espèces de la Chênaie à Charme sont présentes dans les Hêtraies des Picos de Europa.

A côté des Hêtraies, l'espèce s'installe dans beaucoup de groupements liés à une humidité assez élevée : Châtaigneraies, haies de Corylus avellana. Son affinité pour les groupements forestiers est évidente. Néanmoins, on peut trouver des immatures sur micromammifères, en milieux largement ouverts, à partir de 800 mètres d'altitude, notamment dans des prairies méso-hygrophiles.

Les hôtes, en ce qui concerne les adultes, sont représentés en grande partie par le bétail, dont les migrations saisonnières assurent très largement la diffusion des tiques. Le chamois doit jouer un rôle dans la distribution d'Ixodes ricinus en altitude.

Les immatures parasitent divers micromammifères. A côté du très ubiquiste mulot (66 exemplaires parasités) dont le parasitisme est particulièrement fréquent, remarquons l'infestation possible d'Arvicola terrestris, rarement cité dans les listes récapitulatives des hôtes d'Ixodes ricinus, parfois pourtant fort longues. D'autre part, il faut souligner que si le parasitisme larvaire peut affecter un nombre élevé d'animaux dans un biotope donné (parfois la totalité des animaux capturés), le parasitisme par les nymphes est particulièrement faible, peut-être à cause de la date tardive. Le nombre de larves qui parasitent chaque animal est très variable (de 1 à 30), mais le nombre moyen, qui se situe autour de 3 , est relativement faible.

2) Dermacentor marginatus. - Cette espèce a une vaste amplitude écologique dans les Alpes françaises, puisqu'on la trouve depuis l'étage méditerranéen jusqu'à l'étage montagnard. De même, sur les flancs sud des Picos, nous l'avons trouvée depuis l'altitude de 300 mètres (Série du Chêne-vert), jusqu'à l'altitude de 1200 mètres, où nous l'avons identifiée, en même temps qu'Ixodes ricinus dans des éboulis où ne persistait qu'une végétation très clairsemée de noisetiers et d'aulnes. Nous manquons d'informations pour les régions supérieures.

L'espèce paraît peu fréquente en milieu forestier, lorsque le couvert est particuiièrement dense. Elle manifeste, par contre, une affinité pour les milieux ouverts : landes à Papilionacées (Ulex notamment) et à Ericacées, haies de Corylus avellana. De plus, cette espèce paraît, en première analyse, avoir une affinité spéciale pour les formations clairiérées dépendant de Quercus petraea ou de $Q$. tauza qui relaie $Q$. pubescens dans les régions atlantiques. 
Le faible nombre de biotopes découverts et le faible nombre d'animaux parasités dans les biotopes positifs sont vraisemblablement dus à la date tardive des investigations.

3) Dermacentor reticulatus. - Cette espèce était déjà connue d'Espagne (Arthur, 1960, 1963), mais sans précision de lieu. Nous la signalons de Galice, mais il ne nous a pas été possible de vérifier son abondance.

Notons que cette localisation dans l'Ouest espagnol complète parfaitement la zone présomptive de distribution de cette espèce, telle que l'a établi Immler (1973) en se fondant sur des données climatologiques.

\section{B) Tiques endophiles.}

Remarquons d'abord la faible abondance relative des tiques endophiles: sur une cinquantaine de biotopes, c'est seulement une douzaine qui nous ont fourni des tiques endophiles. Cela, pourtant, n'est pas pour nous étonner, parce que nous connaissons par ailleurs la distribution très irrégulière de ces parasites (cf. notamment pour Ixodes trianguliceps, Aeschlimann et al., 1970). D'autre part, le nombre d'animaux capturés dans chaque biotope est parfois insuffisant pour être certain de l'absence du parasite.

Le nombre des biotopes positifs est à peu près le même pour chacune des deux espèces découvertes: Ixodes trianguliceps et Ixodes acuminatus; l'un de ces biotopes leur est commun (Riberas).

Rappelons que la présence en Espagne de ces deux espèces n'a pas encore été signalée. Elles existent donc dans l'Ouest espagnol, tout comme elles existent dans beaucoup d'autres régions de l'Espagne, et nos prochaines publications permettront d'avoir une première vue d'ensemble sur leur répartition dans la Péninsule Ibérique.

I) Ixodes tRianguliceps. - Cette espèce, d'abord considérée comme exclusivement orophile en France, semble. en fait présente partout en France, sauf dans le Midi méditerranéen, tant en plaine qu'en montagne. Beaucournu (1965) a depuis longtemps montré l'abondance de cette espèce à basse altitude, dans de nombreuses régions de France, notamment dans le domaine atlantique ; Aeschlimann (op. cit.), de son côté, a montré pour la Suisse qu'il en était de même dans le domaine médio-européen ; nous avons fait nous-mêmes les mêmes constatations, dans le Sud-Est de la France notamment.

Aussi la capture de cette espèce dans quelques biotopes de basse altitude ne nous a-t-elle pas surpris; il s'agit, notamment, de secteurs où l'influence atlantique était manifeste (proximité de l'Océan). Cependant, le plus grand nombre de biotopes colonisés par cette espèce se situe à partir de 1200 mètres. 
L'écologie d'Ixodes trianguliceps s'apparente, très souvent, à celle d'Ixodes ricinus : en France, on les trouve souvent ensemble dans les mêmes biotopes (Chênaies à Charme, Hêtraies). De fait, c'est l'association sur hôte que nous avons retrouvée le plus fréquemment, soit dans les Châtaigneraies de basse altitude, soit, plus encore, dans les Hêtraies. Cependant, Ixodes trianguliceps paraît pratiquement absent en milieu ouvert, à basse altitude; de plus, en milieu forestier, sa présence paraît moins constante que celle d'Ixodes ricinus. A partir de 1200 mètres, nous l'avons trouvé en milieu ouvert, mais dans des biotopes capables d'assurer une hygrométrie suffisante: éboulis, anciennes galeries d'extraction minière colonisées par les rongeurs.

Le pourcentage d'animaux parasités, dans les biotopes positifs, nous a semblé singulièrement faible par rapport à ce que nous observons couramment dans les Alpes ou même les régions basses avoisinantes (cf. Annexe : liste des captures), sans que nous puissions fournir une explication.

Les hôtes parasités par cette espèce sont classiques.

Soulignons la présence, dans la litière d'un de nos pièges, d'un mâle, fait que nous constatons parfois lors de nos piégeages en France.

2) Ixodes acuminatus. - Cette espèce, dont le déterminisme précis d'installation, dans un biotope donné, est parfois difficile à définir, peut être considérée, jusqu'à plus ample information, comme une espèce atlantico-méditerranéenne. Ainsi, nous ne l'avons jamais trouvée dans les Alpes françaises du Nord.

Son hôte principal est représenté par la belette, sur laquelle on trouve le plus souvent les femelles, mais de très nombreuses espèces de micromammifères peuvent être parasitées par chacun des stades du parasite.

S'il est difficile de donner une vue précise de l'écologie de cette espèce, vu le petit nombre de biotopes découverts, nous soulignerons cependant plusieurs faits :

a) Ixodes acuminatus a été trouvé à basse altitude dans des stations directement soumises à l'influence océanique: la végétation des biotopes indique en général une assez forte hygrométrie: peupleraie humide; haie de noisetier (avec un accompagnement de plantes méso-hygrophiles et mésophiles: Eupatorium cannabinum, Mercurialis perennis, Hedera helix, Glechoma hederaceum, Aspidium sp.) ; bords de ruisseaux.

b) Dans les Picos, cette espèce n'a été trouvée qu'à basse altitude et uniquement en versant sud. Nous ne l'avons jamais décelée dans les formations boisées qui abritent I. ricinus et $I$. trianguliceps. C'est peut être une faible tolérance pour le froid qui la fait disparaître des formations d'altitude.

c) Par contre, lorsqu'une influence plus continentale se fait sentir, comme c'est le cas dans la Sierra de la Cabrera, Ixodes acuminatus est présent à une altitude de 1000 mètres. 
d) Du point de vue des hôtes, Talpa caeca et Arvicola sapidus sont, à notre connaissance, des hôtes nouveaux pour cette espèce.

\section{Conclusion}

Rappelons qu'un tel travail ne saurait donner qu'une idée fragmentaire de la faune ixodidienne dans le Nord-Ouest de l'Espagne. Même si l'on ne se proposait qu'un inventaire des stades immatures des diverses espèces, on devrait, par suite d'une grande variété d'éthologie et de comportement trophique, s'appuyer sur d'autres techniques que la collecte sur micromammifères: recherche au «drapeau», collectes sur oiseaux, grands mammifères...

Deux espèces, fréquentes sur micromammifères, n'ont, d'autre part, pas été décelées par notre étude: Ixodes ventalloi et Rhipicephalus turanicus; il est bien entendu impossible, dans l'état actuel de nos connaissances, de postuler leur absence, même dans les régions prospectées. Tout au plus peut-on penser, d'après ce qu'on connaît de leurs exigences écologiques, qu'elles sont effectivement absentes des Picos de Europa, du moins à partir de l'étage montagnard. 
ANNEXE

Liste des captures

Ixodes trianguliceps

\begin{tabular}{|c|c|c|c|c|c|c|c|c|}
\hline \multirow[b]{2}{*}{ Lieu } & \multirow[b]{2}{*}{ Date } & \multirow[b]{2}{*}{ Altitude } & \multicolumn{2}{|c|}{ Hôtes } & \multicolumn{4}{|c|}{ Stades } \\
\hline & & & Espèces & $\mid \begin{array}{l}\text { Proportion } \\
\text { d'animaux } \\
\text { parasités }\end{array}$ & $\mathrm{F}$ & M & 1 & $\mathrm{n}$ \\
\hline Celada (S) $\ldots \ldots \ldots \ldots$ & $17-9-73$ & $1000 \mathrm{~m}$ & A.S. & $1 / 11$ & 2 & $1^{*}$ & 1 & \\
\hline Fuente de (S) (Teleferico) & $19-9-73$ & $1800 \mathrm{~m}$ & M.N. & $4 / 19$ & 2 & & & 6 \\
\hline $\begin{array}{c}\text { Riano (LE) (Puerto del } \\
\text { ponton) } \ldots \ldots \ldots \ldots \ldots \ldots\end{array}$ & $21-9-73$ & $1300 \mathrm{~m}$ & C.G. & $1 / 2$ & & & 1 & 1 \\
\hline Arenas de Cabrales $(O)$ & $21-9-73$ & $550 \mathrm{~m}$ & A.S. & $2 / 17$ & 1 & & 3 & 1 \\
\hline Lago Enol $(\mathrm{O}) \ldots \ldots \ldots$ & $21-9-73$ & $1050 \mathrm{~m}$ & A.S. & $2 / 12$ & 1 & & 2 & \\
\hline Lago de la Ercinia (O) & $22-9-73$ & $1200 \mathrm{~m}$ & A.S. & $2 / 31$ & & & 4 & \\
\hline Riberas (O) (sortie est) & $25-9-73$ & $120 \mathrm{~m}$ & A.S. & $5 / 33$ & 4 & $1 *$ & 2 & \\
\hline & & xodes ac & uminatus & & & & & \\
\hline Celada (S) $\ldots \ldots \ldots \ldots$ & $17-9-73$ & $880 \mathrm{~m}$ & Arv. S. & $1 / 1$ & & & & 1 \\
\hline Turieno (S) $\ldots \ldots \ldots \ldots$ & $18-9-73$ & $300 \mathrm{~m}$ & A.S. & $2 / 2$ & & & 2 & 1 \\
\hline $\begin{array}{r}\text { Riberas (O) } \\
\text { sortie est) }\end{array}$ & $25-9-73$ & $120 \mathrm{~m}$ & A.S. & $5 / 33$ & 3 & & 3 & \\
\hline Riberas (même biotope) & » & 》 & Must. N. & $1 / 1$ & 1 & & & \\
\hline $\begin{array}{c}\text { Riberas (biotope } 2 \text { : sor- } \\
\text { tie nord) } \ldots \ldots \ldots \ldots \ldots\end{array}$ & 》 & 》 & A.S. & $2 / 8$ & & & & 1 \\
\hline Riberas (même biotope) & $\gg$ & 》 & S.A. & $1 / 5$ & & & & 2 \\
\hline Tablizo $(\mathrm{O}) \ldots \ldots \ldots \ldots$ & $26-9-73$ & & Must. N. & $1 / 1$ & 1 & & & \\
\hline $\begin{array}{c}\text { Ribadelago de Franco } \\
\text { (ZA) (biotope } 1)\end{array}$ & $31-9-73$ & $1000 \mathrm{~m}$ & A.S. & $1 / 6$ & 1 & & 1 & 1 \\
\hline $\begin{array}{c}\text { Ribadelago de Franco } \\
\text { (biotope 2) }\end{array}$ & $2-10-73$ & $》$ & T.C. & $1 / 2$ & & & & 1 \\
\hline
\end{tabular}

* A Appartenant à l'espèce indiquée dans la colonne précédente. 
Ixodes ricinus

\begin{tabular}{|c|c|c|c|c|c|c|c|c|}
\hline \multirow[b]{2}{*}{ Lieu } & \multirow[b]{2}{*}{ Date } & \multirow[b]{2}{*}{ Altitude } & \multicolumn{2}{|c|}{ Hôtes } & \multicolumn{4}{|c|}{ Stades } \\
\hline & & & Espèces & $\begin{array}{l}\text { Proportion } \\
\text { d'animaux } \\
\text { parasités }\end{array}$ & $\mathrm{F}$ & M & 1 & $\mathrm{n}$ \\
\hline $\begin{array}{c}\text { Matamorosa (S) (Rio Iza- } \\
\text { rilla) } \ldots \ldots \ldots \ldots \ldots \ldots \ldots\end{array}$ & $17-9-73$ & $850 \mathrm{~m}$ & A.S. & $1 / 5$ & & & 3 & \\
\hline Arroyal (S) (biotope 1) & $17-9-73$ & $1000 \mathrm{~m}$ & A.S. & $1 / 4$ & & & 2 & \\
\hline Arroyal (S) (biotope 2) & $\gg$ & $1000 \mathrm{~m}$ & A.S. & $2 / 10$ & & & 2 & \\
\hline Celada (S) $\ldots \ldots \ldots \ldots$ & $\gg$ & $1000 \mathrm{~m}$ & A.S. & $2 / 11$ & & & 3 & \\
\hline Turieno (S) $\ldots \ldots \ldots \ldots$ & $18-9-73$ & $300 \mathrm{~m}$ & A.S. & $1 / 2$ & & & & 1 \\
\hline Las Ilces (S) .......... & $19-9-73$ & $850 \mathrm{~m}$ & A.S. & $3 / 6$ & & & 6 & \\
\hline Fuente de (S) $\ldots \ldots \ldots \ldots$ & $20-9-73$ & $1050 \mathrm{~m}$ & vache & & 1 & & & \\
\hline Fuente de (S) (biotope 1) & $19-9-73$ & $1050 \mathrm{~m}$ & A.S. & $2 / 9$ & & & 2 & \\
\hline Fuente de (S) (biotope 2) & $»$ & $1200 \mathrm{~m}$ & libre & & & & & 1 \\
\hline $\begin{array}{c}\text { Fuente de (S) (même bio- } \\
\text { tope) } \quad \ldots \ldots \ldots \ldots \ldots \ldots \ldots\end{array}$ & » & $\gg$ & A.S. & $9 / 23$ & & & 17 & \\
\hline Fuente de (S) (biotope 3) & » & » & A.S. & $4 / 5$ & & & 15 & \\
\hline Arenas de Cabrales (O) & $21-9-73$ & $550 \mathrm{~m}$ & libre & & 4 & & & \\
\hline $\begin{array}{c}\text { Arenas de Cabrales (O) } \\
\text { (même biotope) } \ldots . . .\end{array}$ & $》$ & 》 & A.S. & $5 / 17$ & & & 4 & \\
\hline Arenas $(\mathrm{O})$ (biotope 2) & $»$ & $600 \mathrm{~m}$ & chèvre & & 2 & & & \\
\hline $\begin{array}{c}\text { Covadonga }(\mathrm{O}) \\
\ldots \\
{ }^{\prime}\end{array}$ & 22-9-73 & $180 \mathrm{~m}$ & $\begin{array}{l}\text { A.S. } \\
\text { homme, } \\
\text { non fixée }\end{array}$ & $6 / 8$ & 1 & & 10 & \\
\hline $\begin{array}{c}\text { Route } \text { Covadonga - Lago } \\
\text { Enol } \ldots \ldots \ldots \ldots \ldots \ldots\end{array}$ & » & $400 \mathrm{~m}$ & A.S. & $7 / 8$ & & & 52 & \\
\hline$\gg$ & 》 & $850 \mathrm{~m}$ & A.S. & $3 / 4$ & & & 11 & \\
\hline $\begin{array}{c}\text { Lago Enol (O) (bioto- } \\
\text { pe } 1) \ldots \ldots \ldots \ldots \ldots \ldots\end{array}$ & $21-9-73$ & $1050 \mathrm{~m}$ & A.S. & $8 / 12$ & & & 22 & \\
\hline$\gg$ & $»$ & $\gg$ & E.Q. & $1 / 1$ & & & 1 & \\
\hline Lago Enol (biotope 2) .. & $»$ & $1100 \mathrm{~m}$ & Arv. $\mathrm{T}$. & $1 / 3$ & & & 1 & \\
\hline
\end{tabular}

** Appartenant à l'espèce indiquée dans la colonne précédente. 


\begin{tabular}{|c|c|c|c|c|c|c|c|c|}
\hline \multirow[b]{2}{*}{ Lieu } & \multirow[b]{2}{*}{ Date } & \multirow[b]{2}{*}{ Altitude } & \multicolumn{2}{|c|}{ Hôtes } & \multicolumn{4}{|c|}{ Stades } \\
\hline & & & Espèces & $\begin{array}{l}\text { Proportion } \\
\text { d'animaux } \\
\text { parasités } * *\end{array}$ & F & M & 1 & $\mathrm{n}$ \\
\hline $\begin{array}{cl}\text { Lago } & \text { Enol (O) (biotope } \\
\text { 3) } & \cdots \cdots \cdots \cdots \cdots \cdots\end{array}$ & $21-9-73$ & $1100 \mathrm{~m}$ & A.S. & $5 / 11$ & & & 8 & \\
\hline Lago de la Ercinia $(\mathrm{O})$. & $22-9-73$ & $1200 \mathrm{~m}$ & A.S. & $1 / 31$ & & & 1 & \\
\hline $\begin{array}{c}\text { Riano (LE) (Puerto del } \\
\text { Ponton) } \ldots \ldots \ldots \ldots \ldots\end{array}$ & $21-9-73$ & $1300 \mathrm{~m}$ & A.S. & $1 / 3$ & & & 1 & \\
\hline \multicolumn{9}{|c|}{ Dermacentor marginatus } \\
\hline Turieno $(\mathrm{S}) \ldots \ldots \ldots \ldots$ & $17-9-73$ & $300 \mathrm{~m}$ & libre & & 1 & & & \\
\hline $\begin{array}{c}\text { Fuente de (S) (biotope 2) } \\
\Downarrow\end{array}$ & $19-9-73$ & $\begin{array}{l}1050 \mathrm{~m} \\
1200 \mathrm{~m}\end{array}$ & $\begin{array}{l}\text { A.S. } \\
\text { A.S. }\end{array}$ & $\begin{array}{l}2 / 19 \\
3 / 7\end{array}$ & & & 1 & 2 \\
\hline $\begin{array}{c}\text { Cobas (OR) }(3 \mathrm{~km} \mathrm{~N} \text { de } \\
\text { Cea) } \\
\ldots \ldots \ldots \ldots \ldots \ldots \ldots \ldots\end{array}$ & $30-9-73$ & $600 \mathrm{~m}$ & A.S. & $1 / 24$ & & & & 1 \\
\hline Cobas (même biotope) ... & $》$ & $》$ & libres & & 2 & 1 & & \\
\hline $\begin{array}{c}\text { Cobas }(\mathrm{OR})(6 \mathrm{~km} \mathrm{~N} \mathrm{de} \\
\text { Cea }) \\
\ldots \ldots \ldots \ldots \ldots \ldots\end{array}$ & $》$ & $\gg$ & A.S. & $1 / 4$ & & & & 1 \\
\hline $\begin{array}{c}\text { Cobas (OR) }(8 \mathrm{~km} \mathrm{~N} \text { de } \\
\text { Cea) } \quad \cdots \cdots \cdots \cdots \cdots \cdots \cdots\end{array}$ & $》$ & $\gg$ & A.S. & $2 / 8$ & & & 1 & 1 \\
\hline $\begin{array}{l}\text { Ribadelago de Franco } \\
\quad(\mathrm{ZA}) \text { (biotope } 2) \ldots .\end{array}$ & $2-10-73$ & $1000 \mathrm{~m}$ & A.S. & $1 / 15$ & & & & 1 \\
\hline \multicolumn{9}{|c|}{ Dermacentor reticulatus } \\
\hline $\begin{array}{c}\text { Cobas (OR) }(3 \mathrm{~km} \mathrm{~N} \text { de } \\
\text { Cea) } \quad \ldots \ldots \ldots \ldots \ldots \ldots\end{array}$ & $30-9-73$ & $600 \mathrm{~m}$ & libre & & 1 & & & \\
\hline
\end{tabular}

** Appartenant à l'espèce indiquée dans la colonne précédente.

Légende: - Provinces espagnoles: S: Santander; LE: Leon; O: Oviedo ; OR: Orense ; ZA : Zamora.

- Espèces animales: A.S.: Apodemus sylvaticus; C.G.: Clethrionomys glareolus; M.N.: Microtus nivalis; Arv. S.: Arvicola sapidus; Arv. T. : Arvicola terrestris; S.A. : Sorex araneus ; T.C.: Talpa caeca; Must. N.: Mustela nivalis; E.Q.: Eliomys quercinus.

- Stades: F: femelle; M: mâle; 1 : larve; n: nymphe.

\section{REMERCIEMENTS}

Nous remercions très vivement tous les participants de cette mission, dont l'aide a été déterminante: M $^{\text {He }}$ M.-L. Zbinden, MM. J.-C. Beaucournu, C. Dufour, J.-C. Praz, ainsi que M. G. Lelièvre pour son assistance technique. 


\section{Bibliographie}

ACedo (C. S.), Vericad (J. R.), 1973. - Ectoparasitos de mamiferos y aves del alto Aragon. Rev. Iber. Parasitol., 33, 29-38.

Aeschlimann (A.), Buttiker (W.), Diehl (P.), Eichenberger (G.), Immler (R.), Weiss (N.), 1970. - Présence d'Ixodes trianguliceps (Birula, 1895) et d'Ixodes apronophorus (Schulze, 1924) en Suisse (Ixodoidea ; Ixodidae). Rev. Suisse Zool., 77, 527-536.

ARTHUR (D. R.), 1960. - A Monograph of the Ixodoidea. Pt. V. The genera Dermacentor, Anocentor, Cosmiomma, Margaropus and Boophilus. Cambridge University Press, édit., New York, 251 p.

ARTHUR (D. R.), 1963. - British Ticks, London, Butterworths, 213 p.

Beaucournu (J.-C.), Robert (Y.), 1965. - Description du mâle d'lxodes acuminatus Neumann, 1901. Bull. Mus. Hist. Nat. Paris, 37, 444-449.

Blanc (G.), Bruneau (J.), 1958. - Apparition saisonnière des Ixodes sur lapins, petits mammifères et lézards de la forêt de Nefifik. Arch. Inst. Pasteur Maroc, 5, 493-498.

Emberger (L.), Gaussen (H.), Kassias (M.), de Philippis (A.), 1962. — Carte bioclimatique de la région méditerranéenne. UNESCO, F.A.O., I.G.N.

Emberger (L.), Gaussen (H.), Kassias (M.), de Philippis (A.), 1962. - Carte de la végétation de la région méditerranéenne. Notice explicative. UNESCO, F.A.O., I.G.N.

Gaussen (H.), 1957. - Les ensembles écologiques de la Péninsule Hispanique. P. Inst. Biol. Apl., 26, 9-17.

Gil Collado (J.), 1936. - Acaros Ixodoideos de Cataluna y Baleares. Mus. Cien. Nat. Barcelona, 10, $11 \mathrm{p}$.

GIL Collado (J.), 1938. - Los Acaros (Ixodoideos) de Espana. Datos actuales respecto a su distribucion. Broteria, Sor. Cienc. Nat., 7, 99-109.

GIl Collado (J.), 1948. - Acaros Ixodoideos de Espana. Rev. Sanid. Hig. publ. Madrid, 22, 389-440.

Gilot (B.), Pautou (G.), Moncada (E.), Ain (G.), 1975. - Première contribution à l'étude écologique d'Ixodes ricinus (Linné, 1758) (Acarina, Ixodoidea) dans le sud-est de la France. Acta Tropica, 32, 232-258.

Gilot (B.), Pautou (G.), Moncada (E.), 1976. — L'analyse de la végétation appliquée à la détection des populations de tiques exophiles dans le sud-est de la France : l'exemple d'Ixodes ricinus (Linné, 1758). Acta Tropica, 32, 139-145.

IMMLER (R.), 1973. - Untersuchungen zur biologie und okologie der zecke Dermacentor reticulatus (Fabricius, 1794) (Ixodidae) in einem endemischen vorkommensgebiet. Bull. Soc. Ent. Suisse, 46, 1-70.

Lascombes (G.), 1944. - La végétation des Picos de Europa. Bull. Soc. Hist. Nat. Toulouse, 79, 1-20.

Morel (P.-C.), 1965. - Présence en France de Exopalpiger trianguliceps (Birula, 1893) (Acariens, Ixodoidea). Ann. Parasitol. Hum. Comp., 40, 240-242.

Simon (P.-H.), 1974. - Epidémiologie de la Piroplasmose bovine en Brionnais. Essai d'un plan de Chimioprévention. Thèse médecine vétér., Lyon, $110 \mathrm{p}$.

Valenti (J.-V.), 1968. - La péninsule ibérique. P.U.F. édit., Paris, 290 p. 